\title{
Study on the Estimation of Rock Rheological Parameters under Multi-level Loading and Unloading Conditions
}

\author{
Han Bing, Fu Qiang \\ China National Institute of Standardization, 100191, Beijing, China
}

\begin{abstract}
Determining reasonable rheological constitutive models based on laboratory rock creep test data and estimating rheological parameters are important means of studying rheological mechanical property of rocks. Previous rock uniaxial or triaxial compression creep tests mostly adopted the method of multi-level loading. In order to study the influence of unloading stress on the estimation of rock rheological parameters, rock triaxial creep tester RLW-2000 was used in this paper to carry out triaxial compression creep tests for granites in a given area with two different stress paths of multi-level loading and stepwise unloading. Study results indicate that rock rheological parameters obtained vary with different stress paths. The instantaneous elastic modulus and viscosity coefficient obtained through loading show a stepwise increase, while the viscoelastic modulus shows a progressive decrease.
\end{abstract}

\section{Introduction}

Rheological property is an important mechanical property of rocks. It is closely related to long-term stability of geotechnical engineering. Determining reasonable rheological constitutive models based on laboratory rock creep test data and estimating rheological parameters are important means of studying rheological mechanical property of rocks. Lots of references have mentioned the study on uniaxial and triaxial compression creep tests of rocks under loading conditions in recent years[1-6], but there are few studies on rock creep tests and rheological mechanical property under unloading conditions. Practice indicates that engineering rock masses are inherently different under two different stress conditions of loading and unloading. In many rock mass projects including underground caverns, rock slopes and rock foundations, the load or surrounding rock stress imposed on the rock mass is generally released in a stepwise manner as the construction progresses. It is always in the stress environment of progressive unloading. The rock creep under such stepwise unloading conditions is an important physical and mechanical property and engineering phenomenon of rock masses. If corresponding rock mechanical parameters are obtained based on traditional loading mechanical test results only, the data are often greatly different from actual observation results. Therefore, carrying out rock creep tests under unloading conditions and studying estimates of corresponding rheological mechanical parameters have great theoretical value and actual engineering significance.

In this paper, with surrounding rocks of underground storage to be built in a coastal area as the object of study, laboratory triaxial compression creep tests were respectively carried out for granite specimens in the storage with two different stress paths of multi-level loading and stepwise unloading. Test results were used to identify the rheological constitutive model, thus obtaining the change law of rheological mechanical parameters under two stress conditions of loading and unloading.

\section{Rock Triaxial Creep Test}

Rock triaxial creep test is an important means to study rheological mechanical property of rocks. Test results are the base of rheological constitutive model study. Actual test data are of great importance to the inversion of known rheological model parameters and identification of unknown rheological models. Considering that engineering rock masses are always under threedimensional stress conditions, rock triaxial creep tests were carried out in this paper for the purpose of fully learning about rheological mechanical property of surrounding rocks of the underground storage.

\subsection{Test equipment}

Rock triaxial rheological tester RLW-2000 was used to carry out laboratory creep tests. This tester consists of an axial loading system, a confining pressure loading system, a servo system, a control system, a data acquisition and automatic drawing system and so on. Fig. 1 shows the tester.

Control parts of axial and confining pressure loading systems adopt EDC which allows force control or deformation control, also supports smooth switching between the control methods during the test. The pressure stabilizing system uses an AC servo motor for automatic 
pressure stabilizing. The deformation measuring device uses displacement extensometers from the American Teratech Corporation, including two sets: axial and radial extensometers, with the relative error of $\pm 0.5 \%$.

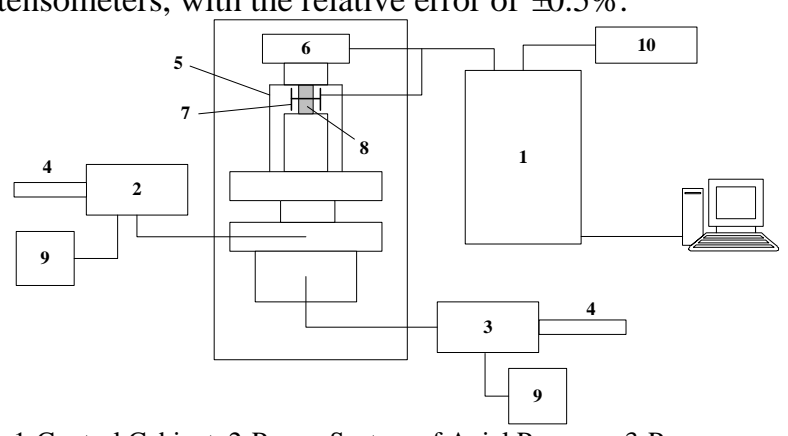

1-Control Cabinet, 2-Power System of Axial Pressure, 3-Power System of Confining Pressure, 4-Ball Screw, 5-Pressure Cell, 6Pressure Sensor, 7-Deformation Sensor, 8-Rock Core, 9-Servo Motor, 10-Power Supply

Figure 1. RLW-2000 triaxial rheological test system of rock.

\subsection{Test conditions and plans}

Rock cores used in the test were all taken from the project site. Rocks in the storage are mainly medium- and coarse-grained syengranites of the overbridge unit in the early cretaceus period of the mesozoic erathem, with good appearance integrity and uniformity. Three typical places were determined on site for drilling and sampling analysis. Rock cores were made into cylindrical standard specimens in a unified way, with a diameter of $55 \times 110$ $\mathrm{mm}$. The method of triaxial creep test was adopted for temperature-controlled long-lasting test. Well-prepared rock cores were sealed with oil-proof corrosion-resistant shrinkable tubes and placed in the self-balancing pressure chamber of the rock axial creep tester. With the central position well adjusted, the axis of the specimen coincided with the loading center line of the tester, avoiding nonhomogeneity of rock loading resulting from eccentric compression. The test was carried out in a special creep laboratory under constant temperature and humidity conditions. Temperature in the laboratory was strictly controlled at $25 \pm 0.1^{\circ} \mathrm{C}$ during the test. The computer collected the data of load, deformation and time automatically during the test, and displayed the axial strain-time relation curve in real time.

In order to fully consider the influence of different stress paths on rock rheological property and mechanical parameters, two plans were designed for this test: multilevel loading and stepwise unloading.

Plan (1): The confining pressure keeps constant, while the axial pressure uses the method of multi-level loading, namely, based on the instantaneous breaking strength obtained from conventional triaxial compression test, divide the maximum load to be imposed into several levels, and then impose the load on the same specimen gradually from the lowest level to the highest level. The imposing duration of the load at each level should depend on the strain rate or stress rate variation of the sample. Conduct low-stress pre-compression for the specimen first, and observe the displacement continuously. Generally, the pre-compression period is controlled within about 24 hours. Then impose the preset confining pressure on the specimen through the hydraulic system at a loading rate of $0.05 \sim 0.02 \mathrm{MPa} / \mathrm{s}$, to make the specimen under hydrostatic pressure. When the confining pressure reaches the set value and deformation becomes stable, impose axial load at a rate of $500 \mathrm{~N} / \mathrm{s}$. When the load reaches stress of the first level, keep the axial stress unchanged, observe and record the relationship between axial strain and time. After the creep deformation of the specimen becomes stable, impose the axial load of the next level, and maintain the stress of this level imposed on the specimen constantly. Repeat the above steps, and stop the test until the rheodestruction of the specimen under the preset stress of the last level.

Plan (2): The confining pressure keeps constant, and the axial pressure uses the method of stepwise unloading. Divide the maximum load to be imposed into several levels, and then decrease the axial load on the same specimen level by level from high to low. In order to avoid that excessive primary load causes the specimen to be damaged instantaneously or enter the accelerated creep stage ahead of time, set the stress of the first level to be $80 \%$ of instantaneous breaking strength, keep the load of the level unchanged, observe and record the relationship between axial strain and time, and conduct the first level of unloading for the level of stress after the creep deformation of the specimen becomes stable. The load decrement is the same with the load increment in the loading test. Stabilize the level of stress and keep it unchanged after the preset load of the next level is reached, until the start of the next level of unloading. The determination of duration for each level of load is as same as that in Plan (1). As granite specimens used in the test are hard brittle rocks whose rheological characteristics are not obvious under low stress, the test should be stopped when the axial load decreases to 40MPa.

\subsection{Analysis of test results}

Fig. 2 and 3 respectively show triaxial creep test curves of specimens 3-26 taken from Drill No. 3 under two stress path conditions of stepwise unloading and multilevel loading. Unloading test was carried out first. It can be seen from Fig. 2 that reversible deformation occurred rapidly on specimens at the moment of unloading, indicating an instantaneous elastic recovery of rocks. Hysteretic rebound deformation, i.e. elastic aftereffect, happened over the time. The specimen had instantaneous strain at the moment when each level of stress was released. The strain capacity increased with the decrease of stress level. Under most levels of stress, instantaneous strain accounted for the main part of overall deformation. For the loading test in Fig. 3, the deformation-time curve of the specimen shows that the creep value under low stress was relatively low; deformation became stable after a short initial deformation; the creep deformation rate of rocks decreased gradually with the time under relatively high stress; finally deformation tended to be restrained and entered the stage of steady flow; under the stress of the last level, rock deformation was aggravated sharply, 
showing a tendency of obvious accelerated creep. Cracks appeared outside the specimen and expanded quickly. Rapid evolution and accumulation of damage finally resulted in rock rupture.

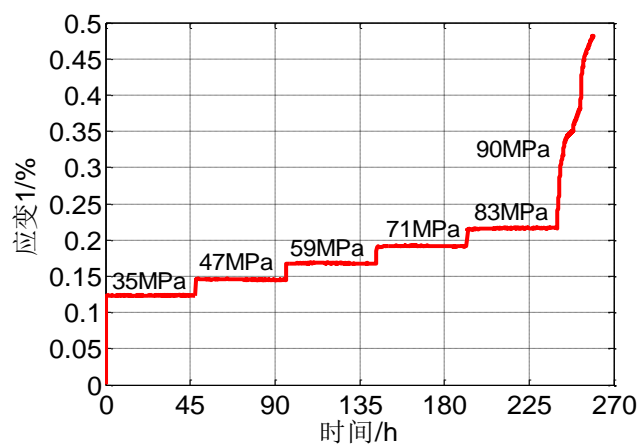

Figure 2. Triaxial creep curve of rock under step unload.

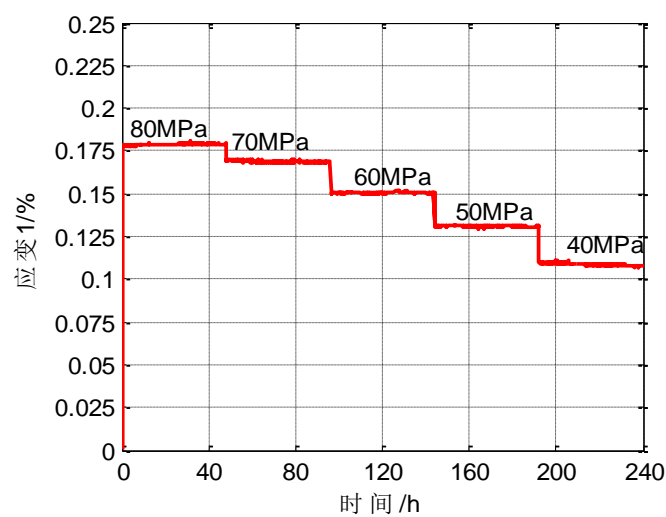

Figure 3. Triaxial creep curve of rock under step load.

\section{Model Identification and Parameter Estimation}

To carry out rheological study on rocks, it is important to select proper rheological models according to laboratory rock creep test data and determine corresponding rheological parameters through parameter inversion or curve fitting. Rheological constitutive models of rocks often fall into three types: empirical formula, combined model and integral model, among which combined model consisting of elastic, viscous and plastic basic elements are most widely used.

\subsection{Rheological model identification}

Rheological characteristics of high-strength rock materials like granites are not obvious under low stress. According to Fig. 2 and 3, strain tended to be restrained gradually with the time under most levels of stress; both the elastic strain that recovered instantaneously and elastic aftereffect happened at the moment of unloading, with rheological property conforming to the feature reflected by the generalized Kelvin model. Therefore, the three-parameter generalized Kelvin model was used to describe the mechanical property, as shown in Fig. 4, with the constitutive equation as below:

$$
\varepsilon=\frac{E_{0}+E_{1}}{E_{0} E_{1}} \sigma-\frac{\eta_{1}}{E_{0}} \dot{\varepsilon}+\frac{\eta_{1}}{E_{0} E_{1}} \dot{\sigma}
$$

Wherein, $E_{0}$ and $E_{1}$ respectively refer to instantaneous elastic modulus and viscoelastic modulus while $\eta_{1}$ is viscosity coefficient.

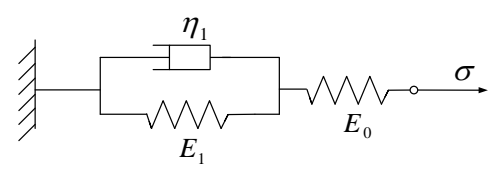

Figure 4. H-K rheological model.

\subsection{Methods of parameter estimation}

Presently, methods used to determine rock rheological parameters according to laboratory test data and curves mainly include regression analysis[7], optimized separation[8], least square method[9-10], curve decomposition[11-12] and so on. In this paper, the method of optimized separation for viscoelastic parameters put forward in Reference[8] was used to calculate rheological parameter. Firstly, designate the design variable and objective function. Take the parameter to be inverted as the design variable. Calculate the strain residual sum of squares based on the sum of $n$ test data $\varepsilon$ from the creep test data, and take the minimum value as the objective function for optimized separation of parameters. So, the design variable is

$$
X=\left(E_{0}, E_{1}, \eta_{1}\right)=\left(X_{1}, X_{2}, X_{3}\right)
$$

The objective function is

$$
f(X)=\sum_{i=1}^{n}\left[\varepsilon\left(t_{i}\right)_{c}-\varepsilon\left(t_{i}\right)_{m}\right]^{2}
$$

Wherein, $\varepsilon\left(t_{i}\right)_{c}$ and $\varepsilon\left(t_{i}\right)_{m}$ respectively refer to the strain value obtained through calculation and testing at the time of $t_{i}$.

For the generalized Kelvin model, the objective function is

$$
f(x)=\sum_{i=1}^{n}\left[\frac{\sigma}{E_{1}}+\frac{\sigma}{E_{2}}\left(1-\exp \left(-\frac{E_{2}}{\eta_{1}} t_{i}\right)\right)-\varepsilon\left(t_{i}\right)_{m}\right]^{2}
$$

In order to simplify the above optimization problem, possible upper and lower limit estimates of the design variable can be provided according to analogies of engineering experience, thus to establish the following constraint condition

$$
a_{i} \leq X_{i} \leq b_{i} \quad(i=1,2,3,4)
$$


Wherein, $X_{i}$ is the No. $i$ design variable; $b_{i}$ and $a_{i}$ are the upper limit and lower limit of $X_{i}$ respectively. With the mathematical model established based on Formula (2), (4) and (5), the method of multi-constraint optimization can be used to approach the optimal point $X^{l}=\left(E_{1}, E_{2}, \eta_{1}\right)$ step by step and solve the minimization of the objective function. In this case, $X^{l}$ is the rock characteristic parameter vector to be solved. According to the above method, determine rheological parameters as shown in Table 1:

Table 1. Rheological parameters.

\begin{tabular}{|c|c|c|c|c|}
\hline \multirow{2}{*}{$\begin{array}{c}\text { Test } \\
\text { plans }\end{array}$} & \multirow{2}{*}{$\begin{array}{c}\text { Sress/ } \\
\mathrm{MPa}\end{array}$} & \multicolumn{3}{|c|}{ Rheological parameters } \\
\cline { 3 - 5 } & $E_{0}(\mathrm{MPa})$ & $E_{1}(\mathrm{MPa})$ & $\left.\eta_{1}(\mathrm{Mpa})\right)$ \\
\hline \multirow{4}{*}{ loading } & 35 & $4.655 \times 10^{4}$ & $8.479 \times 10^{5}$ & $1.033 \times 10^{7}$ \\
\cline { 2 - 5 } & 47 & $4.708 \times 10^{4}$ & $6.713 \times 10^{5}$ & $3.812 \times 10^{7}$ \\
\cline { 2 - 5 } & 59 & $4.792 \times 10^{4}$ & $5.256 \times 10^{5}$ & $4.987 \times 10^{7}$ \\
\cline { 2 - 5 } & 71 & $5.381 \times 10^{4}$ & $3.970 \times 10^{5}$ & $5.663 \times 10^{7}$ \\
\cline { 2 - 5 } & 83 & $5.944 \times 10^{4}$ & $2.142 \times 10^{5}$ & $7.012 \times 10^{7}$ \\
\cline { 2 - 5 } & mean & $4.697 \times 10^{4}$ & $5.312 \times 10^{5}$ & $4.501 \times 10^{7}$ \\
\cline { 2 - 5 } $\begin{array}{c}\text { unloadin } \\
\text { g }\end{array}$ & 80 & $5.454 \times 10^{4}$ & $3.097 \times 10^{5}$ & $5.663 \times 10^{7}$ \\
\cline { 2 - 5 } & 70 & $4.603 \times 10^{4}$ & $4.784 \times 10^{5}$ & $3.214 \times 10^{7}$ \\
\cline { 2 - 5 } & 60 & $3.866 \times 10^{4}$ & $5.930 \times 10^{5}$ & $2.547 \times 10^{7}$ \\
\cline { 2 - 5 } & 50 & $3.727 \times 10^{4}$ & $7.415 \times 10^{5}$ & $1.189 \times 10^{7}$ \\
\cline { 2 - 5 } & 40 & $3.649 \times 10^{4}$ & $8.293 \times 10^{5}$ & $0.680 \times 10^{7}$ \\
\cline { 2 - 5 } & mean & $3.840 \times 10^{4}$ & $5.904 \times 10^{5}$ & $2.659 \times 10^{7}$ \\
\hline
\end{tabular}

\subsection{Analysis of test results}

It can be seen from Table 1 that for rheological parameters estimated based on the multi-level loading creep curve, the obtained instantaneous elastic modulus $E_{0}$ and viscosity coefficient $\eta_{1}$ show a tendency of progressive increase with the increase of stress, while the viscoelastic modulus $E_{1}$ decreases gradually; for rheological parameters obtained through the stepwise unloading creep curve, the instantaneous elastic modulus $E_{0}$ and viscosity coefficient $\eta_{1}$ show a tendency of progressive decrease with the decrease of stress, while the viscoelastic modulus $E_{1}$ increases gradually. The parameter change law is exactly the opposite of that of parameters under loading conditions. According to the comparison of the means of all rheological parameters obtained under two different stress paths, the parameter $E_{0}$ and $\eta_{1}$ obtained under loading conditions are $81.75 \%$ and $59.08 \%$ of relevant parameters under unloading conditions, while for the parameter $E_{1}$, the mean under unloading conditions is $89.97 \%$ of that under loading conditions. This shows that the two different stress paths of multi-level loading and stepwise unloading have a certain influence on rock rheological mechanical property and parameter estimation. In previous rock uniaxial or triaxial creep tests, rheological parameters were mostly estimated based on the multi-level loading creep curve. However, for rock mass projects featured by excavation, the rock stress and strain redistribution law obtained from numerical calculation based on such rheological mechanical parameters cannot reflect actual conditions of the project truly and completely.

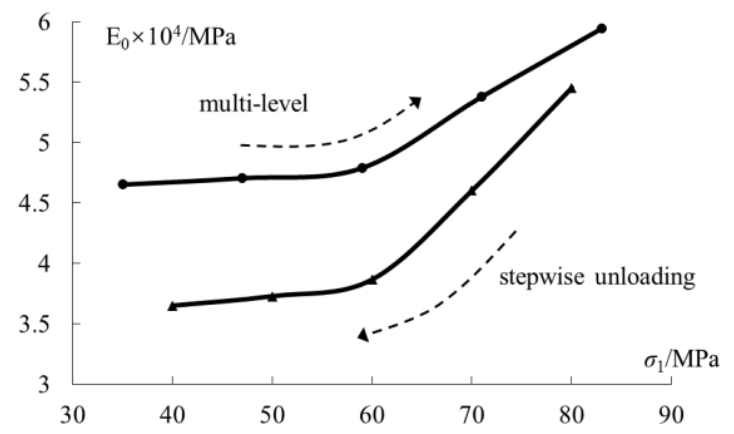

Figure 5. The relation between the elastic modulus E0 and stress paths.

Figure 6. The relation between the viscoelastic modulus E1 and

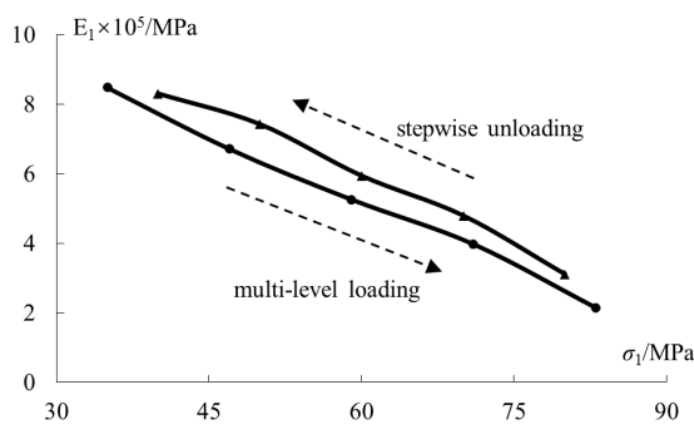

stress paths.

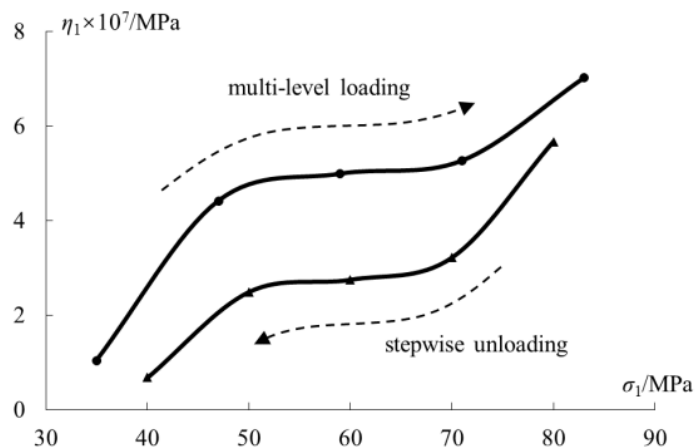

Figure 7. The relation between the viscous coefficient $\eta 1$ and stress paths.

\section{Conclusions}

Rock rheological constitutive model identification and parameter estimation are important issues in rock mechanical theory and engineering practice. In this paper, rock mechanical experimental study was carried out for granites in a certain area under two different stress path conditions of multi-level loading and stepwise unloading based on laboratory rock triaxial compression creep tests. On the basis of the creep curve in two stress states, the method of optimized separation was used to estimate rock rheological mechanical parameters, and the following 
conclusions were drawn through the analysis of test and calculation results:

(1) According to Fig. 5 and 7, Rheological parameters $E_{0}$ and $\eta_{1}$, obtained based on the multi-level loading creep curve, show a tendency of progressive increase with the increase of stress, while the viscoelastic modulus $E_{1}$ decreases gradually, as shown in Fig. 6; parameters $E_{0}$ and $\eta_{1}$ obtained through the unloading creep curve show a tendency of progressive decrease with the decrease of stress, while the viscoelastic modulus $E_{1}$ increases gradually.

(2) The comparison of means shows a great difference in rheological parameters obtained under loading and unloading conditions. Therefore, for the long-term stability analysis of specific rock mass excavation projects, if the numeric calculation is carried out based on rock rheological parameters obtained through loading tests, it can hardly reflect the change law of rocks in actual unloading environment. Hence, unloading creep tests should be carried out to analyze rheological property of rocks, so as to obtain corresponding mechanical parameters.

\section{References}

1. G. Liu and Y. S. Zhou: Seismology and Geology. Rheology of Felsic Rocks and Relative Influence Factors, Vol. 34 No.2, (2012), p. 365-383

2. J. Sun: Rock rheological mechanics and its advance in engineering applications. Chinese Journal of Rock Mechanics and Engineering, Vol. 26 No.6 (2007), p.1081-1115

3. W. H. Gao, Q. N. Chen, and Z. Y. Huang: Study on the creep damage constitutive model of soft rocks considering rheological softening and intelligent identification of the parameters. China Civil Engineering Journal, Vol. 45 No.2 (2012), p.104-110

4. G. J. Wang, L. Zhang, and Y. W. Zhang: Experimental investigation on the creep-damage- rupture characteristics of salt rock. Engineering Mechanics, Vol. 30 No.4 (2013), p.288-293

5. Z. W. Yang, A. B. Jin, and Y. T. Gao: Nonstationary Nishihara model in the particle flow code. Chinese Journal of Engineering, Vol. 37 No.7 (2015), p.831838

6. T. Xu, C. A. Tang, and J. Zhao: Modelling the timedependent rheological behaviour of heterogeneous brittle rocks. Geophysical Journal International, Vol. 189 No.3 (2012), p. 1781-1796

7. C. Z. Pu, P. Cao, C. Y. Zhang, and Q. C. Zhang: Variable Parameters Nonlinear Creep Damage Model of Rock with Consideration of Aging, Engineering Mechanics. Vol. 34 No.6 (2017), p. 1727

8. Z.M. Shi and L. Zhang: Study on Rheological Test of Jinping Greenschist Under Step Load. Journal of Tongji University (Natural Science). Vol. 39 No.3 (2011), p. 320-326

9. M. F. Wang, B. Hu, H. F. Jiang, G. J. Ou, and L. Gan: Experiment and model investigation on shear rheological mechanical properties of granite. Journal of Central South University (Science and Technology). Vol. 45 No.9 (2014), p. 3111-3120

10. Y. Y. Huang and H. Zheng: Preliminary study of equivalent rheological model for jointed rock. Rock and Soil Mechanics, Vol. 32 No.12 (2011), p. 35663570

11. X. G. Wang, B. Hu, B. Q. Lian, J. L. Yan, Z. J. Xu, and Z. H. Zhao: Modified nonlinear viscoelasticplastic rheological model and parameter identification of shear rheological model for granite. Chinese Journal of Geotechnical Engineering, Vol. 36 No.5 (2014), p. 916-921

12. S. G. Zhang, W. B. Liu, and Y. T. Wang: Study on Non-stationary Creep and Parameter Determination of Granite. Journal of Disaster Prevention and Mitigation Engineering, Vol. 37 No.3 (2017), p. 435441 